\title{
Positron Annihilation in Liquid Scintillator for Electron Antineutrino Detection
}

\author{
Yasushi Kino, ${ }^{*, a}$ Tsutomu Sekine, ${ }^{\text {a }}$ Yasuyoshi Sato, ${ }^{\text {a }}$ Hiroshi Kudo, ${ }^{a}$ Fumihiko Suekane, ${ }^{b}$ \\ Atsuto Suzuki, ${ }^{\mathbf{b}}$ Yasuo Ito, ${ }^{\mathrm{c}}$ and Takenori Suzukid ${ }^{\mathrm{d}}$ \\ ${ }^{a}$ Department of Chemistry, Graduate School of Science, Tohoku University, Sendai 980-8578, Japan \\ ${ }^{\mathrm{b}}$ Research Center for Neutrino Science, Graduate School of Science, Tohoku University, Sendai 980-8578, Japan \\ ${ }^{\mathrm{c}}$ Research Center for Nuclear Science and Technology, The University of Tokyo, Tokai, Ibaraki 319-1195, Japan \\ ${ }^{\mathrm{d}}$ Radiation Science Center, High Energy Accelerator Research Organization (KEK), Tsukuba, Ibaraki 305-0801, \\ Japan
}

\section{Received: May 16, 2000; In Final Form: August 21, 2000}

\begin{abstract}
Positron annihilation processes in a liquid scintillator to be used for the detection of electron antineutrinos in the KamLAND project were studied. The liquid scintillator was a mixture of isoparaffin, pseudocumene and 2,5diphenyloxazole. We measured positron annihilation lifetime (PAL) spectra which contained important timing information on identification of the electron antineutrinos. The PAL spectra of a nitrogen-saturated liquid scintillator consisted of three lifetime components. Two fast components with the lifetime of $0.19 \pm 0.05 \mathrm{~ns}$ and $0.48 \pm$ $0.05 \mathrm{~ns}$ correspond to the mixture of para-positronium annihilation and free positron annihilation. The delayed component $(3.41 \pm 0.02 \mathrm{~ns})$ with the intensity of $48.9 \pm 0.3 \%$ is ascribed to pick-off annihilation of ortho-positronium ( $o$-Ps) annihilation. To understand the $2 \gamma$ annihilation processes of $o$-Ps in the liquid, we examined the effects of dissolved gases (nitrogen, oxygen, helium and argon) and mixing ratio of isoparaffin and pseudocumene which were the main components of the liquid scintillator. The lifetime of $o$-Ps was drastically shortened in the presence of oxygen, but not for other gases. The reaction rate constant for quenching of $o$-Ps by oxygen was obtained from the $\mathrm{O}_{2}$ concentration dependence of $o$-Ps lifetime component. The pick-off annihilation of $o$-Ps with the liquid scintillator is explained based on the bubble model. We discuss a possible microscopic picture of the surroundings of Ps in the liquid scintillator.
\end{abstract}

\section{Introduction}

Recently an epoch making discovery has been made in elementary particle physics. Namely evidence for neutrino oscillation was observed by a large water Cherenkov detector called SuperKamiokande.' This observation is the first clue to a theory beyond the standard model of elementary particles, giving excitement to physicists. There are three kinds of neutrinos; an electron-type $\left(v_{\mathrm{e}}\right)$, a muon-type $\left(v_{\mu}\right)$ and a tautype $\left(v_{\tau}\right)$. What they observed was the oscillation of $v_{\mu}$.

Another large neutrino detector, called KamLAND (Kamioka Liquid scintillator AntiNeutrino Detector), is under construction next to SuperKamiokande. ${ }^{2}$ A main purpose of KamLAND experiments is to look for oscillation of electrontype neutrino by detecting electron antineutrinos $\left(\bar{v}_{e}:\right.$ antiparticle of $v_{e}$ ) generated in nuclear reactors located around 170 $\mathrm{km}$ away. This experiment is fully sensitive to a set of oscillation parameters, so called MSW Large Angle Solution (for example, see Reference 3, further references therein), which gives an answer to the puzzle of solar neutrino deficit, and has a good chance to observe the oscillation of electron-type neutrinos. To compensate for the very small reaction cross sections of neutrinos, the KamLAND detector makes use of 1,000 tons of a liquid scintillator (a mixture of paraffin oil, pseudocumene and DPO (2,5-diphenyloxazole)) as the active target. The $\bar{v}_{e}$ reacts with a proton in the liquid scintillator to produce a positron and a neutron,

$$
\bar{v}_{e}+p \rightarrow e^{+}+n
$$

A typical energy of the positron is a few $\mathrm{MeV}$ and the positron annihilates with a surrounding electron within a few ns, producing two $511 \mathrm{keV} \gamma$ rays,

*Corresponding author. E-mail: kino@mail.cc.tohoku.ac.jp. FAX: +88-22-217-6597.

$$
e^{+}+e^{-} \rightarrow 2 \gamma
$$

The neutron is thermalized quickly, and then absorbed by a proton after $170 \mu$ s of lifetime, producing a $2.2 \mathrm{MeV} \gamma$ ray,

$$
n+p \rightarrow d+\gamma
$$

The $\bar{v}_{e}$ is identified by observing a coincident event of both the positron-related signals (eq 1,2) and the deuteron signal (eq 3 ). It is important to know the time structure of positron annihilation, because the coincidence measurement is sensitive to the positron annihilation lifetime (PAL) in a detector. Moreover, this information can be used to distinguish positron signals from other instantaneous background signals, such as $\beta$ rays from natural radioactive substances contained in the liquid scintillator.

Some of the positrons injected and stopped in bulk liquid annihilate with the surraunding electrons directly, while other positrons form positronium (Ps) which is a neutral bound state of a positron and an electron. Positronium is the lightest of the hydrogen-like atoms. Positronium has two substates depending on the total spin angular momentum $S$. The spin singlet state $(S=0)$ is called para-positronium ( $p$-Ps) which annihilates emitting two mono energetic $511 \mathrm{keV} \gamma$ rays with the lifetime of $0.125 \mathrm{~ns}$. The spin triplet state $(S=1)$ is called ortho-positronium (o-Ps) which annihilates emitting three $\gamma$ rays with the lifetime of $140 \mathrm{~ns}$ in vacuum. However, in condensed matters the lifetime of $o$-Ps is reduced to several ns, due to interactions with surrounding electrons emitting two mono energetic $511 \mathrm{keV} \gamma$ rays. The processes involved are a "pick-off", a "chemical reaction" and a "spin conversion". The intensity and the lifetime of Ps strongly depend on the physical and chemical conditions of the bulk liquid. Over the past few decades, a number of studies have been made on the positron annihilation in nonpolar organic solutions (for example, see Reference 4, further references therein). Generally, a lifetime 
of positron in nonpolar organic liquids comprises two short components and one or more long components. Approximately, the two short lifetimes lie in the range of $0.1-0.5 \mathrm{~ns}$, and the long lifetime is a few ns. The intensity of the long lifetime component ranges from 5 to $50 \%$. Structures of the lifetime spectrum depend on liquid species, and are changed by dissolution of oxygen. ${ }^{5-10}$ Quenching of $o$-Ps by oxygen was first found in liquid argon and nitrogen by Paul. ${ }^{5}$ Kerr et al. ${ }^{6}$ reported an angular correlation between the two annihilation $\gamma$ rays. Their experimental data showed that the quenching was associated with the pick-off process. Systematic lifetime measurements for various organic liquids were carried out by Lee and Celitans. ${ }^{8}$ They claimed that the chemical reaction between $o$-Ps and $\mathrm{O}_{2}$ (or NO) is a diffusion-controlled process.

In this paper, we examine the time structure of positron annihilation in the liquid scintillator to be used for the $\bar{v}_{e}$ detection. We also investigate dependence of the PAL spectra on gaseous species such as oxygen, nitrogen, helium and argon dissolved in it, and discuss a $o$-Ps formation process in the liquid scintillator.

\section{Experimental}

The PAL spectra were obtained by a conventional fast-fast coincidence system with plastic scintillators, having a time resolution of $0.3 \mathrm{~ns}$ at FWHM. A ${ }^{22} \mathrm{Na}$ positron source $(0.6$ $\mathrm{MBq}$ ) was sandwiched between two sheets of $7.5 \mu \mathrm{m}$ polyimide (Kapton) film where $15 \%$ of positrons annihilated with a single lifetime component ( $\tau=0.38 \mathrm{~ns}$ ). The total counts of each measurement was about $10^{7}$. The lifetime measurements were performed at room temperature $\left(23^{\circ} \mathrm{C}\right)$ in pseudocumene (PC: COSMO) from Matsuyama Peteroleum Co., isoparaffin (IP: PARAOL250) from Showa Shell Sekiyu and mixtures of PC and IP solutions. In addition, the lifetime was measured for a liquid scintillator (LS), to be used for the $\bar{v}_{e}$ detection, composed of $80 \%$ of IP, $20 \%$ of PC and $2 \mathrm{~g} \mathrm{~L}^{-1}$ of 2,5diphenyloxazole (DPO) from Dojindo Laboratories. The samples were not degassed as in References 4-10. Because the IP used here is a mixture of various fractions having different boiling points, it was difficult to degas the sample without causing a change in the initial composition. The positron source was dipped in the scintillation liquid $(15 \mathrm{~mL})$ in a glass vial. The liquid was saturated with gases (oxygen, nitrogen, helium, argon or a mixture of nitrogen and oxygen) by bubbling under the atmospheric pressure. The glass vial was tightly capped and sealed in a vinyl bag filled with the same gas, and then subjected to the lifetime measurement.

An appropriate mixing ratio of nitrogen and oxygen was attained by using a gas mixer (GB-2B, KOFLOC. Co.), and the concentrations of nitrogen and oxygen in the liquid were determined by gas chromatography. The pure gas concentrations in the liquid calculated by applying the Henry's law using the published gas solubilities ${ }^{11}$ are listed in Table 1.

Doppler broadening spectra were taken with a pure germanium solid state detector equipped with a pulse hight analyser in the energy region around $511 \mathrm{keV}$ during the life time measurements. An FWHM value for $\gamma$ ray of $514 \mathrm{keV}$ from ${ }^{85} \mathrm{Sr}$ was determined as $0.755 \mathrm{keV}$.

TABLE 1: Gas Concentration $\left(10^{21}\right.$ molecules $\left.\mathrm{L}^{-1}\right)$ in Organic Liquids Used

\begin{tabular}{lllll}
\hline & $\mathrm{N}_{2}$ & $\mathrm{O}_{2}$ & $\mathrm{He}$ & $\mathrm{Ar}$ \\
\hline IP & 3.5 & 5.5 & 0.59 & 0.54 \\
PC & 2.8 & 4.1 & 0.53 & 6.0 \\
LS & 3.4 & 5.2 & 0.58 & 1.6 \\
\hline
\end{tabular}

\section{Results and Discussion}

The accumulated PAL data were analyzed using a program PATFIT $^{12}$ with three components of the lifetime. The $\chi^{2}$ per degree of freedom of each analysis was about 1.0. The lifetimes of the components were about $0.2,0.5$ and $3 \mathrm{~ns}$, respectively. The first two components represent mixing of lifetimes of the $p$-Ps and free positron annihilation in the bulk liquid. The third component with a long exponential-tail separated from the other components represents $2 \gamma$-annihilation caused by interactions between $o$-Ps and bulk liquid.

Figure 1 shows PAL spectra observed for the LS saturated with $\mathrm{N}_{2}$ and $\mathrm{O}_{2}$. The lifetime of $o$-Ps appearing in the tail region of the spectra was drastically shortened in the presence of oxygen. In Table 2 , the lifetimes $\left(\tau_{i}\right)$ and intensity $\left(I_{i}\right)$ after correcting for the components corresponding to the positrons annihilating in the source material are listed for the $\mathrm{N}_{2}$ and $\mathrm{O}_{2}$ saturated samples. In the case of $\mathrm{N}_{2}$ saturation, the values in Table 2 give a time structure of positron annihilation corresponding to the $\bar{v}_{e}$-injected LS for the KamLAND project. The $o$-Ps intensity $I_{3}$ did not change by dissolution of $\mathrm{O}_{2}$ and $\mathrm{N}_{2}$, indicating that neitrher $\mathrm{O}_{2}$ nor $\mathrm{N}_{2}$ would affect the Ps formation process in the LS.

The measured lifetimes and intensities of the $o$-Ps component are summarized in Table 3. All but oxygen gas dissolution in the liquid scintillators examined here hardly affected the $o$-Ps lifetime $\tau_{3}$.

An effect of $\mathrm{O}_{2}$ concentration on the $o$-Ps annihilation rate $\left(\lambda_{3}=\tau_{3}^{-1}\right)$ is shown in Figure 2 for the concentration from $10^{-3}$ to $10^{-2} \mathrm{M}$. The $\lambda_{3}$ linearly increases with increasing $\mathrm{O}_{2}$ concentration, indicating the presence of chemical reaction of $o$-Ps with $\mathrm{O}_{2}$ molecule in the annihilation process. Therefore the annihilation rate $\lambda_{3}$ should be expressed as

$$
\lambda_{3}=\lambda_{3 \gamma}+\lambda_{\text {liquid }}+\kappa\left[\mathrm{O}_{2}\right]
$$

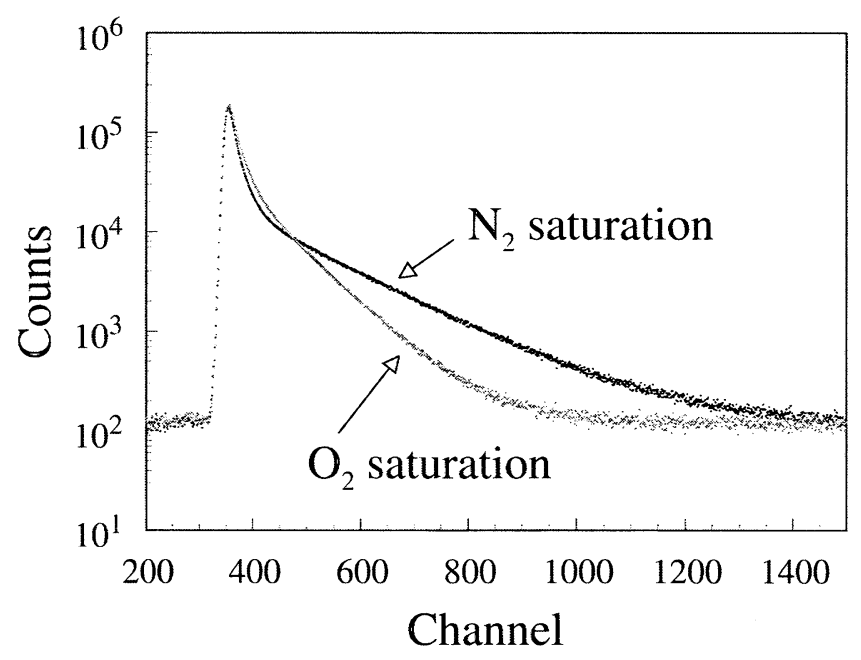

Figure 1. PAL spectra for the liquid scintillator (LS); the black dots for $\mathrm{N}_{2}$ saturation and the gray dots for $\mathrm{O}_{2}$ saturation (1 channel = $0.0253 \mathrm{~ns})$.

TABLE 2: Lifetime and Intensity of the $i$ th Component in PAL Spectra Measured for the Liquid Scintillator (LS)

\begin{tabular}{rccc}
\hline$i$ & 1 & 2 & 3 \\
\hline $\mathrm{N}_{2}$ saturated $\tau_{i} / \mathrm{ns}$ & $0.19 \pm 0.05$ & $0.48 \pm 0.05$ & $3.41 \pm 0.02$ \\
$I_{i} / \%$ & $30 \pm 2$ & $21 \pm 2$ & $48.9 \pm 0.3$ \\
\hdashline $\mathrm{O}_{2}$ saturated $\tau_{i} / \mathrm{ns}$ & $0.21 \pm 0.04$ & $0.49 \pm 0.03$ & $1.83 \pm 0.01$ \\
$I_{i} / \%$ & $21 \pm 2$ & $30 \pm 3$ & $48.9 \pm 0.4$
\end{tabular}


TABLE 3: Lifetime $\tau_{3}$ and Intensity $I_{3}$ of $o$-Ps in Isoparaffin (IP), Pseudocumene (PC) and Liquid Scintillator (LS)

\begin{tabular}{|c|c|c|c|c|c|c|}
\hline & \multicolumn{3}{|c|}{$\tau_{3} / \mathrm{ns}$} & \multicolumn{3}{|c|}{$\tau_{3} / \%$} \\
\hline & IP & $\mathrm{PC}$ & LS & IP & $\mathrm{PC}$ & LS \\
\hline $\mathrm{He}$ & $3.25 \pm 0.01$ & $2.98 \pm 0.01$ & $3.20 \pm 0.02$ & $40.2 \pm 0.2$ & $45.1 \pm 0.4$ & $48.5 \pm 0.3$ \\
\hline $\mathrm{Ar}$ & $3.32 \pm 0.03$ & $3.01 \pm 0.03$ & $3.42 \pm 0.03$ & $40.7 \pm 0.4$ & $45.8 \pm 0.6$ & $49.5 \pm 0.7$ \\
\hline $\mathrm{N}_{2}$ & $3.50 \pm 0.03$ & $3.03 \pm 0.02$ & $3.41 \pm 0.02$ & $39.6 \pm 0.2$ & $46.1 \pm 0.3$ & $48.9 \pm 0.3$ \\
\hline $\mathrm{O}_{2}$ & $1.88 \pm 0.01$ & $1.78 \pm 0.01$ & $1.83 \pm 0.01$ & $40.7 \pm 0.2$ & $44.2 \pm 0.2$ & $49.1 \pm 0.5$ \\
\hline
\end{tabular}

where $\lambda_{3 \gamma}$ is the rate of $3 \gamma$-annihilation of $o$-Ps in vacuum, $\lambda_{\text {liquid }}$ is the rate of pick-off annihilation of $o$-Ps with electrons in the surrounding species, and the third term is the rate of $2 \gamma$-annihilation of $o$-Ps through a chemical reaction with dissolved oxygen. Here, $\kappa$ is the reaction rate constant and $\left[\mathrm{O}_{2}\right]$ is the $\mathrm{O}_{2}$ concentration. The straight lines in Figure 2 obtained by the $\chi^{2}$-fit with eq 4 give the rate constants $\kappa$ as $29.2 \pm 0.3 \mathrm{M}^{-1} \mathrm{~ns}^{-1}$ for LS, $32 \pm 4 \mathrm{M}^{-1} \mathrm{~ns}^{-1}$ for PC and $26.5 \pm 0.6 \mathrm{M}^{-1} \mathrm{~ns}^{-1}$ for IP. These values are consistent with those of Reference 8 ( $\kappa=10$ $\left.\sim 100 \mathrm{M}^{-1} \mathrm{~ns}^{-1}\right)$ and Reference $7\left(\kappa=20 \sim 50 \mathrm{M}^{-1} \mathrm{~ns}^{-1}\right)$. For the pick-off annihilation induced by one of the electrons in molecules, $\lambda_{\text {liquid }}$ is given by

$$
\lambda_{\text {liquid }}=\pi r_{0}^{2} c n Z_{\text {eff }} \text {, }
$$

where $r_{0}$ is the classical radius of electron $\left(r_{0}=\alpha^{2} a_{\text {Bohr }}=2.8 \mathrm{fm}\right)$, $c$ the speed of light, $n$ the number density of molecules and $Z_{\text {eff }}$ the effective number of annihilation electrons per molecules. It is difficult to determine the value $Z_{\text {eff }}$ directly. We assume that $Z_{\text {eff }}$ is represented by the number of valence electrons of a molecule. Because a positron, having positive charge, feels repulsive force from a cation inside the valence orbital, the probability of finding a positron in the inner shell is expected to be small. To calculate $\lambda_{3}$, we used $Z_{\text {eff }}=92$ for IP and $Z_{\text {eff }}=48$ for PC. Ambiguity of the choice of $Z_{\text {eff }}$ will be discussed later. The values of $\lambda_{3}$ calculated with eq 5 were several times larger than observed ones. We recall the bubble model $^{13}$ to explain the overestimation. The bubble model assumes the existence of a space where $o$-Ps is free from pickoff annihilation. A repulsive electron exchange interaction between Ps and the surrounding molecules traps Ps in the free space called a positronium bubble. Because of a strong quantum effect of Ps having the light mass $\left(2 m_{e}\right)$, a part of the wave function of Ps can spread outside the bubble, where the pick-off annihilation occurs. Taking account of this model, we modify the $\lambda_{\text {liquid }}$ of eq 5 multiplying a probability $P(x)$ of finding Ps outside the bubble,

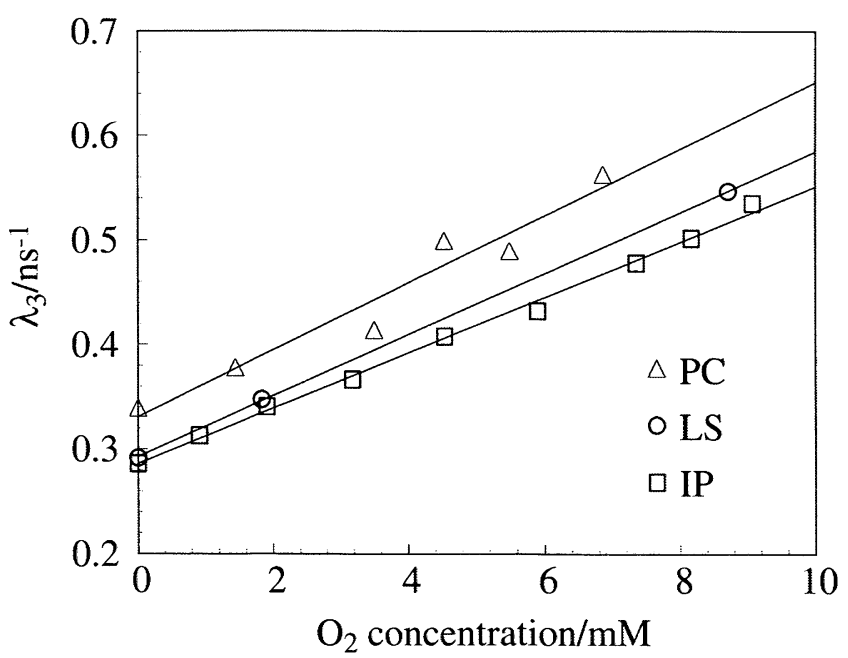

Figure 2. Oxygen concentration dependence of $\lambda_{3}$. Circle, square and triangle stand for PC, LS and IP, respectively. The solid lines show a relation expressed in eq 4 .

$$
\lambda_{\text {liquid }}=\pi r_{0}^{2} c n Z_{\text {eff }} P(x),
$$

where $x$ is a variable depending on the state of the Ps bubble. We assume that Ps is in a three dimensional square-well potential. The zero-point energy $\varepsilon(k r)$ of Ps in this potential is calculated by means of a quantum mechanical procedure,

$$
\varepsilon(k r)=U \sin ^{2}(k r),
$$

where $U$ and $r$ are the depth and the radius of the Ps bubble, respectively. The value of $k$ is the wave number of Ps given by the boundary condition of the wave function at the bubble surface. The total energy $E(r)$ of the Ps bubble is

$$
E(r)=\varepsilon(k r)+4 \pi r^{2} \sigma+\frac{4 \pi}{3} r^{3} p .
$$

The second term represents the surface energy associated with the surface tension $\sigma$ of the bulk liquid and the third term represents the volume energy at pressure $p$. We neglect the third term, the value of which is estimated to be 2 orders of magnitude smaller than the other terms. The Ps bubble stably exists at the stationary point of $E(r)$, namely,

$$
\left.\frac{d}{d r} E(r)\right|_{r=a}=0,
$$

where $a$ is the most probable radius. Using the Ps bubble wave function $\Psi_{k}(r)$, the probability $P(x)$ is given by,

$$
P(x)=\int_{a}^{\infty}\left|\Psi_{k}(r)\right|^{2} r^{2} d r=\frac{\sin ^{2} x}{1-x \cot x},
$$

with

$$
x=k a \text {. }
$$

Here, $x$ is the only one dimensionless variable in the bubble model, and describes all of the physical quantities $(a, E(a)$, $\varepsilon(x), U$ and $\mu)$. Because the probability $P(x)$ is in the range of $0-1$, the variable $x$ is defined in

$$
\frac{\pi}{2} \leq x \leq \pi \text {. }
$$

Using the observed values of $\lambda_{3}, x$ is determined from eqs. 4,6 and 10.

The calculated values of $a, E(a), \varepsilon(x), U$ and $\mu$ are listed in Table 4. The values of $a, \varepsilon(x)$ and $U$ are given as,

$$
\begin{aligned}
a & =\left(\frac{\hbar^{2}}{16 \pi m_{e} \sigma} \frac{x^{3}}{x-\tan x}\right)^{\frac{1}{4}}, \\
\varepsilon(x) & =\hbar \sqrt{\frac{\pi \sigma}{m_{e}} x(x-\tan x)},
\end{aligned}
$$

and

$$
U=\hbar \sqrt{\frac{\pi \sigma}{m_{e}}} \frac{\sqrt{x(x-\tan x)}}{\sin ^{2} x}
$$

A penetration length $\mu$ is estimated by the density of $o$-Ps outside the potential (bubble), 


$$
\left|\Psi_{k}(r \geq a)\right|^{2} \propto \exp \left(-\frac{r}{\mu}\right),
$$

namely,

$$
\mu=-\frac{1}{2}\left(\frac{\hbar^{2}}{16 \pi m_{e} \sigma}\right)^{\frac{1}{4}} \frac{\tan x}{\sqrt[4]{x(x-\tan x)}} .
$$

The number of carbon atoms in an IP molecule is assumed as 15 on an average. The physical quantities listed in Table 4 hardly change against the number of carbon atoms because of the cancellation in the term of $n Z_{\text {eff }}$. The bubble model contains ambiguity of the choice of $Z_{\text {eff }}$, and we examined $Z_{\text {eff }}$ dependence of the physical quantities. The physical quantities summarized in Table 4 were calculated with $Z_{\text {eff }}$ as (a) the number of valence electrons of the molecule, (b) the number of all electrons of the molecule and (c) a half of the valence electrons. Case (b) gives the upper limit of $Z_{\text {eff }}$ and case (c) is for comparison. The radius $a$, energies $\varepsilon(x)$ and $E(a)$ of the Ps bubble are approximately independent of the choice of $Z_{\mathrm{eff}}$, because the values of $a, \varepsilon(x)$ and $E(a)$ change abruptly at $x=\pi / 2$ where $P(x)=1$ and $Z_{\text {eff }}$ has the minimum value, and gradualy change elsewhere. On the other hand, the potential depth $U$ and the penetration length $\mu$ strongly depend on the choice of $Z_{\text {eff }}$.

A positronium can be regarded as a rigid particle in the bubble, because kinetic energy $\varepsilon(x)$ is much smaller than the first excited energy of Ps $(5.1 \mathrm{eV})$. Excitation of the surrounding molecules in the collision with Ps is negligible, because the Ps is light and its momentum transfer is small. A radius of the Ps bubble is about four times larger than that of Ps, and close to a size of the surrounding molecules. The potential of the Ps bubble may be affected by a shape and a configuration of molecules. For further investigation of a Ps bubble the surface tension $\sigma$ is modified by taking account of microscopic information on molecules. The penetration depth is smaller than the size of the surrounding molecule. Positronium in the Ps bubble can contact only a few molecules and a surface effect of the potential well is not negligible.

To examine the effects of dissolved gas on the Ps lifetime, our experimental results were compared with literature values. $^{14}$ In Table 5, the values of $\tau_{3}$ are listed together with the surface tension $\sigma$ and the density $\rho$ of a variety of organic liquids. The probability $P(x)$ of finding Ps outside the bubble is calculated with the relation,

$$
\lambda_{3}=\lambda_{3 \gamma}+\pi r_{0}^{2} c n Z_{\mathrm{eff}} P(x)
$$

From the literature values the probabilities $P(x)$ are given as $0.150 \pm 0.009$ for alkane and $0.161 \pm 0.004$ for aromatic compounds. The probabilities of both $\mathrm{N}_{2}$-dissolved IP and PC show the same tendencies as the degassed samples. Therefore, the use of samples saturated with $\mathrm{N}_{2}$ instead of the degassed samples is a good approximation. Note that the LS is saturated with $\mathrm{N}_{2}$ in the practical use for the $\bar{v}_{e}$ detection to avoid the strong quenching of the luminescence of DPO by oxygen. According to eq 13, the radius of the Ps bubble is inversely proportional to the fourth root of the surface tension of the bulk liquid, because the probabilities $P(x)$ are almost constant for both alkane and aromatic compounds.

The formation of $o-\mathrm{PsO}_{2}$ (an analog of $\mathrm{HO}_{2}$ ) compound would play an important role in rapid quenching of $o$-Ps lifetime in bulk liquids. ${ }^{5}$ The direct spin conversion process $o-\mathrm{Ps}+\mathrm{O}_{2} \rightarrow p-\mathrm{Ps}+\mathrm{O}_{2}$ * should be impossible, because this reaction needs at least $0.977 \mathrm{eV}\left(\mathrm{O}_{2}\left({ }^{3} \Sigma_{g}^{-}\right) \rightarrow \mathrm{O}_{2}\left({ }^{1} \Delta_{g}\right)\right)$ which is much higher than thermal energy. Since the thermalization rate of $o$-Ps is significantly higher than the quenching rate of $o$ Ps by oxygen, the epithermal effect is negligible. A resonant state of $o-\mathrm{PsO}_{2}$ is formed during the collision,

$$
o-\mathrm{Ps}+\mathrm{O}_{2} \rightarrow\left[o-\mathrm{PsO}_{2}\right]^{*}
$$

There are two $2 \gamma$-annihilation processes via the $o-\mathrm{PsO}_{2}$ state. One is the spin conversion process. Because of the strong spin-spin interaction between the $o$-Ps and the $\mathrm{O}_{2}$, the $o$-Ps would change its spin state changing the orbital angular momentum of the resonant state.

TABLE 5: $o$-Ps lifetime $\tau_{3}$ in IP and PC in Comparison with that in Alkane and Aromatic Compounds ${ }^{14}$ Obtained for Degassed Samples

\begin{tabular}{lccll}
\hline & $\tau_{3} / \mathrm{ns}$ & $\sigma / \mathrm{dyn} \mathrm{cm}^{-1}$ & $\rho / \mathrm{g} \mathrm{cm}^{-3}$ & $P(x)$ \\
\hline Alkane & & & & \\
$n$-Pentane & 4.25 & 16.05 & 0.6262 & 0.146 \\
$n$-Hexane & 3.92 & 18.4 & 0.6603 & 0.151 \\
$n$-Heptane & 3.81 & 20.14 & 0.6837 & 0.151 \\
$n$-Octane & 3.49 & 21.62 & 0.6985 & 0.162 \\
$n$-Dodecane & 3.43 & 25.35 & 0.7487 & 0.155 \\
$n$-Tetradecane & 3.35 & 26.56 & 0.7628 & 0.157 \\
Isooctane & 4.05 & 18.77 & 0.6919 & 0.141 \\
2,2-Dimethylbutane & 4.46 & 16.31 & 0.6485 & 0.135 \\
average & & & & $0.150 \pm 0.009$ \\
\hline IP(Thiswork) & 3.50 & 23.10 & 0.787 & 0.150 \\
\hline Aromatic & & & & \\
Benzene & 3.15 & 28.9 & 0.8765 & 0.164 \\
Toluene & 3.24 & 28.52 & 0.8669 & 0.158 \\
Ethylbenzene & 3.02 & 29.29 & 0.867 & 0.168 \\
$o$-Xylene & 3.08 & 30.31 & 0.8801 & 0.162 \\
$m$-Xylene & 3.2 & 29.02 & 0.8642 & 0.159 \\
$p$-Xylene & 3.21 & 28.54 & 0.86104 & 0.159 \\
Mesithylene & 3.21 & 28 & 0.8652 & 0.156 \\
average & & & & $0.161 \pm 0.004$ \\
\hline PC(Thiswork) & 3.03 & 29.71 & 0.8758 & 0.164 \\
\hline & & & &
\end{tabular}

TABLE 4: Physical Quantities of Ps Bubble Calculated with $Z_{\text {eff }}$ Adopting (a) the Number of Valence Electrons in the Molecule, (b) the Number of all of Electrons in the Molecule and (c) the Number of half of Valence Electrons in Isoparaffin (IP) and Pseudocumene (PC)

\begin{tabular}{rllllllll}
\hline & $\mathrm{Z}_{\text {eff }}$ & $x$ & $P(x)$ & $a / \mathrm{nm}$ & $E(a) / \mathrm{eV}$ & $\varepsilon(x) / \mathrm{eV}$ & $U / \mathrm{eV}$ & $\mu / \mathrm{nm}$ \\
\hline (a) IP & 92 & 2.35 & 0.150 & 0.45 & 0.89 & 0.52 & 1.04 \\
PC & 48 & 2.32 & 0.164 & 0.42 & 1.00 & 0.59 & 1.11 & 0.096 \\
- (b) IP & 122 & 2.43 & 0.113 & 0.46 & 0.91 & 0.53 & 1.22 & 0.096 \\
PC & 66 & 2.41 & 0.120 & 0.43 & 1.03 & 0.59 & 1.34 & 0.080 \\
- (c) IP & 46 & 2.14 & 0.361 & 0.41 & 0.82 & 0.52 & 0.74 & 0.149 \\
PC & 24 & 2.10 & 0.330 & 0.38 & 0.93 & 0.60 & 0.80 & 0.151 \\
\hline
\end{tabular}




$$
\left[o-\mathrm{PsO}_{2}\right]^{*} \rightarrow\left[p-\mathrm{PsO}_{2}\right]^{* \prime} \rightarrow p-\mathrm{Ps}+\mathrm{O}_{2},
$$

where $\left[p-\mathrm{PsO}_{2}\right]^{* \prime}$ is another resonant state having different quantum number from $\left[p-\mathrm{PsO}_{2}\right]^{*}$. The other is annihilation with orbital electron in oxygen atoms. Although the resonant state is a short-lived state, $\left[o-\mathrm{PsO}_{2}\right]^{*}$ is stabilized in collision with the surrounding molecule $\mathrm{M}$,

$$
\left[o-\mathrm{PsO}_{2}\right]^{*}+\mathrm{M} \rightarrow\left[o-\mathrm{PsO}_{2}\right]+\mathrm{M}^{*}
$$

We have measured Doppler broadening of the $511 \mathrm{keV}$ annihilation $\gamma$ rays with a pure germanium solid state detector having a resolution of $0.755 \mathrm{keV}$ (FWHM at $514 \mathrm{keV}$ from ${ }^{85} \mathrm{Sr}$ ). The spin conversion process gives a sharp peak profile, because the $p$-Ps described in eq 20 is in thermal energy. On the other hand the pick-off annihilation with orbital electrons gives a broad peak profile, because orbital electrons have a higher momentum $(\sim \mathrm{keV} / \mathrm{c})$. The observed $\gamma$ ray energy spectra had the same FWHM (1.25 keV) and FWTM (2.29 keV) for all of the samples examined here. These results suggest that the quenching of $o$-Ps by oxygen in the LS may be ascribed to the spin conversion process via $o-\mathrm{PsO}_{2}$ compound, and are consistent with the angular correlation reported in Reference 6.

Figure 3 shows the annihilation rate $\lambda_{3}$ and the intensity $I_{3}$ in a mixture of IP and PC saturated with $\mathrm{N}_{2}$ as a function of the PC concentration. The value of $\lambda_{3}$ has linear dependence on the PC concentration having a slope of $(4.8 \pm 0.3) \times 10^{-4} \mathrm{~ns}^{-1} \%{ }^{-1}$. The value of $I_{3}$ steeply increases to a peak around $20 \%$ of PC concentration, and gradually decreases with increasing PC concentration. The Ps intensity depends on the probability of finding excess electrons. This may be explained in terms of anti-recombination effect (see Reference 15 and the references cited therein). This effect involves shallow electron traps that are normally provided by aromatic compounds like PC. In the terminal positron spur the positron competes with the parent ions $\left(\mathrm{M}^{+}\right)$in capturing electrons as,

$$
\begin{aligned}
& e^{+}+e^{-} \rightarrow \mathrm{Ps}, \\
& \mathrm{M}^{+}+e^{-} \rightarrow \mathrm{M}^{*} .
\end{aligned}
$$

When aromatic compounds like PC are added, the electrons are scavenged by aromatic compounds. The electron mobility is suppressed, and hence the recombination of electrons with the positive ions is delayed in time.

$$
e^{-}+\mathrm{PC} \rightarrow \mathrm{PC}^{-}
$$

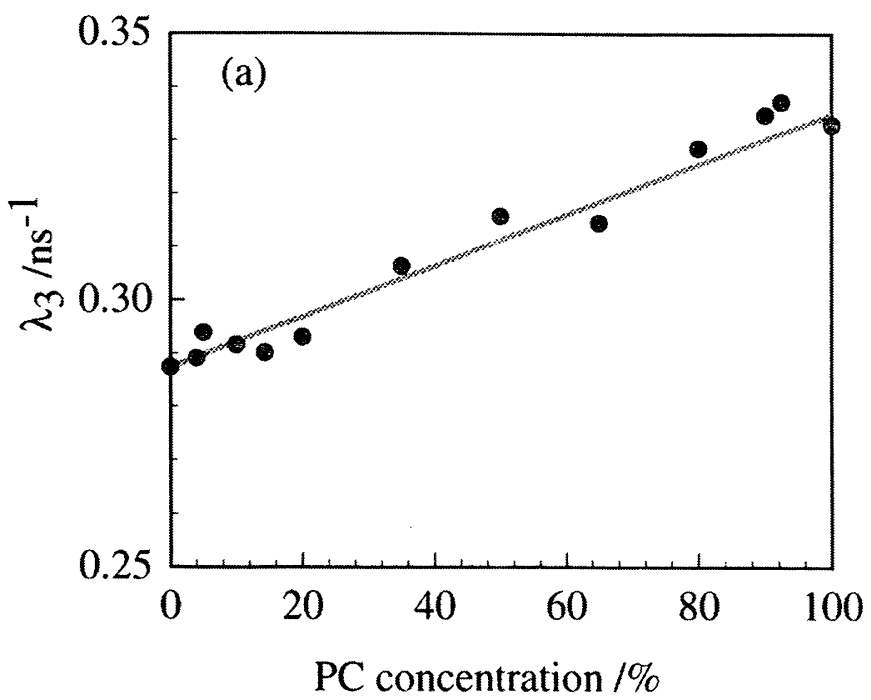

Figure 3. (a) PC concentration dependence of $\lambda_{3}$ in a mixture of IP and PC. circle stands for experimental values and the lines are a guide for eyes.
Meanwhile the positron can pick up the shallowly-trapped electron.

$$
e^{+}+\mathrm{PC}^{-} \rightarrow \mathrm{Ps}+\mathrm{PC}
$$

In this way PC interrupts recombination process of (23) and effectively enhances the Ps formation. This effect is called anti-recombination. It is known that many aromatic compounds that can provide shallow traps of electrons show the anti-recombination effect. ${ }^{15}$ The increase of the Ps intensity is saturated at PC concentration of about $20 \%$, and gradually decreases at higher concentrations. It is not easy to explain this gradual decrease in a straightforward way since many factors, like the number of excess electrons, the spur size, the electron and positron mobilities and so on, may change at the wide range of composition of IP and PC.

In conclusion, the measured time structure of positron annihilation in the liquid scintillator for electron antineutrino detection are described as follows. The lifetimes (intensities) of two fast compornents are $0.19 \pm 0.05 \mathrm{~ns}(30 \pm 2 \%)$ and $0.48 \pm 0.05$ ns $(21 \pm 2 \%)$ corresponding to the mixture of $p$-Ps annihilation and free positron annihilation. The lifetime (intensity) of the delayed component is $3.41 \pm 0.02 \mathrm{~ns}(48.9 \pm 0.3 \%)$ for $o$ Ps annihilation. The lifetime of the third component is close to the transit time spread (3.6 ns at FWHM) of photomultiplier which is to be used for KamLAND projects. The measured reaction rate constant for quenching of $o$-Ps by oxygen in the liquid scintillator was $29.2 \pm 0.3 \mathrm{M}^{-1} \mathrm{~ns}^{-1}$. The pick-off annihilation of $o$-Ps with liquid scintillator is explained based on the bubble model. The radius and the energies estimated for the Ps bubble are approximately independent of the choice of the effective number of annihilation electrons per molecule $Z_{\text {eff. }}$.

Acknowledgments. We thank Professor K. Nagase and Doctor W. Zheng for determination of the gas concentration in the liquid samples using gas chromatography. We also thank Professor M. Kikuchi for the use of a gas mixer and an oxygen analyzer. YK wishes to acknowledge Inoue Foundation for Science for their generous financial support.

\section{References}

(1) Y. Fukuda, et al. (Super-Kamiokande Collaboration), Phys. Rev. Lett. 81, 1562 (1998).

(2) http://www.awa.tohoku.ac.jp/KamLAND/index.html. This web site contains detailed information and present status about KamLAND project.

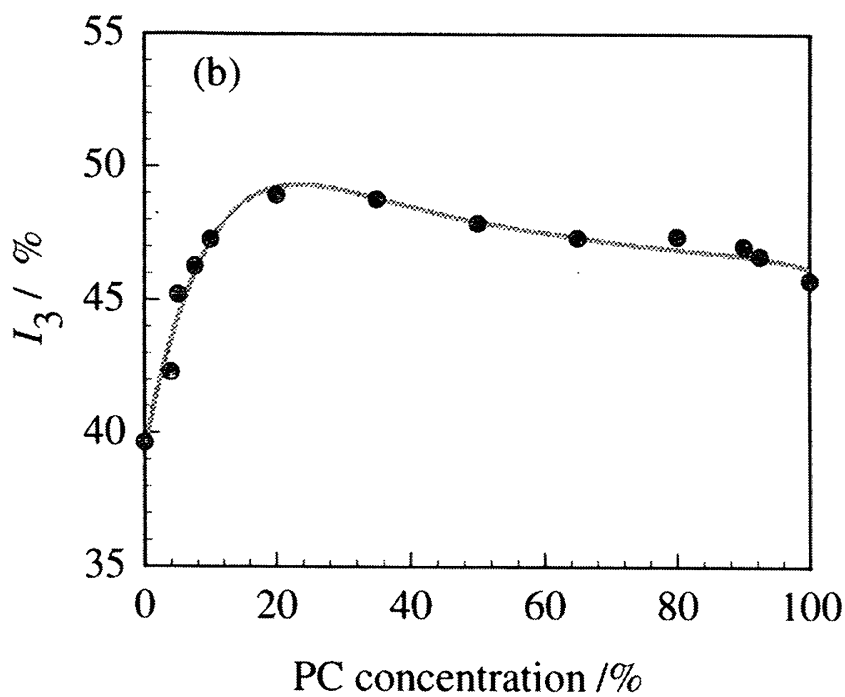

. (b) PC concentration dependence of $I_{3}$ in a mixture of IP and PC. The 
(3) J. N. Bahcall, P. I. Krastev, and A. Yu. Smirnov, Phys. Rev. D60, 093001 (1999).

(4) O. E. Mogensen, Positron annihilation in chemistry (Springer-Verlag, Berlin, 1995).

(5) D. A. L. Paul, Can. J. Phys. 37, 1059 (1959).

(6) D. P. Kerr, A. M. Cooper, and B. G. Hogg, Can. J. Phys. 43, 963 (1965).

(7) J. Lee and G. J. Celitans, J. Chem. Phys. 42, 437 (1965).

(8) J. Lee and G. J. Celitans, J. Chem. Phys. 44, 2506 (1966).

(9) A. M. Cooper, G. J. Laidlaw, and B. Hogg, J. Chem. Phys. 46, 2441 (1967).

(10) P. R. Gray, C. F. Cook, and G. P. Sturm, J. Chem. Phys.
48, 1145 (1968).

(11) A. Seidell and W. F. Linke, Solubilities: inorganic and metal-organic compounds, 4th edition by Atherton Seidell (American Chemical Society, Washington, 1965).

(12) P. Kirkegaard, N. J. Pedersen, and E. Eldrup, PATFIT- 88 (Riso-M-2740).

(13) R. A. Ferrell, Phys. Rev. 110, 1355 (1958).

(14) F. M. Jacobsen, O. E. Mogensen, and N.J. Pedersen, Proc. 8th Int. Conf. Positron Annihilation, Gent, Belgium (1988).

(15) Y. Ito, in Positron and Positronium Chemistry edited by D. M. Schrader and Y. C. Jean (Elsevier Scientific Publishers Co., Amsterdam, 1988). 